\title{
Application of Synchronization Status Message Based on the Self-healing Ring Network with Synchronous Digital Hierarchy
}

\author{
YIN Shu Hua ${ }^{\star^{1, a}}$, LEI Hü, b , TIAN Lu ${ }^{3, c}$ \\ ${ }^{1}$ Xi'an fanyi university, Xi'an, China \\ Shuhua_y@126.com
}

Keywords: Synchronization; Timing-Ring; Multiplex Section Protection; APS; SSM

\begin{abstract}
This paper studies how to avoid timing loop in the self-healing ring (SHR) network with synchronous digital hierarchy (SDH) and analyzes the generation and mechanism of timing loop in $\mathrm{SDH}$ local ring network as well as the harmfulness of timing loop to the network. Based on these results, a new method is proposed to avoid the generation of timing loop in the multiplex section protection ring by combining the K1 and K2 bytes in APS protocol of the multiplex section with S1 byte in the synchronization status message (SSM). This method improves the synchronization performance of SDH SHR network.
\end{abstract}

\section{Introduction}

In the SDH network, since the payload is asynchronously transferred, the different rates in the sender and receiver cause pointer justification, which is supposed to result in the jitter and wander of output signals. The greater jitter is bound to result in the loss of frame (LOF), while the excessive wander is supposed to lead to the sliding of terminal equipments. Therefore, attention has been paid to satisfy the requirements of ITU-T standard for the jitter and wander of the output signals in the SDH network synchronization. In the SDH network, when all the nodes are synchronous, pointer justification events rarely happen; while when the synchronization quality is degraded, these events frequently occur. In turn, multiple pointer justification events bring about stricter requirements for the synchronizer which has a phase smoothing effect on the boundary of SDH/plesiochronous digital hierarchy (PDH). Hence, the synchronous performance of the network is crucial.

By reducing the slip rates and the times of pointer justification, the sliding damage and the influence of jitter and wander on network services are reduced. Besides, Through effectively controlling the sliding, this paper attempts to have all equipments in the network using the reference clock with a same frequency [1.2.3].

\section{Generation of timing loop}

Timing loop refers to the phenomenon that clocks track their output signals directly or indirectly through the network. The SDH equipment clocks (SEC) are able to receive multiple external synchronous signals, which are automatically switched based on the priority. When there is no system safety monitor (SSM), timing loops are easy to be formed. When the timing loop passes through a ring network, synchronization supply units (SSU) are equipped in the places where a timing signal enters into and leaves the $\mathrm{SDH}$ ring network. $\mathrm{SSU}_{1}$ is configured in the entering place of timing into the SDH ring to form a gateway equipment, namely, $\mathrm{SDH}$ equipment timing source (SETS ${ }_{1}$ ). $\mathrm{SSU}_{\mathrm{k}}$ is provided in the leaving place of timing to form $\mathrm{SETS}_{\mathrm{n}}$. As presented in Figure 1, when the main timing path breaks down, timing loops are formed between two network units. At this moment, the clock is gradually skewed, resulting in large frequency offset. As a result, network services are interrupted.

The clock of node locks its signal so that a timing loop is generated. If the reference timings of all node clocks in the ring network are independent, timing loops are impossible to occur. In the usage of local ring network with multi-clock, the timings of all the nodes are from the external network. This scheme has high requirements for the performance of clocks and high economic cost. In addition, 
there occur periodic slides, so this kind of synchronization mode is not applicable in network-connection.

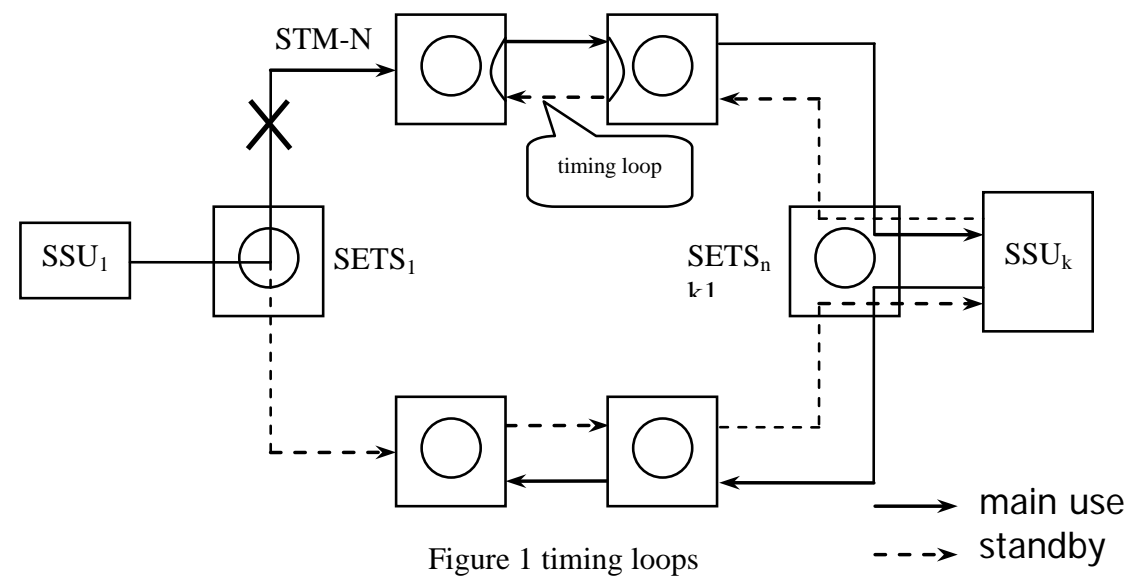

\section{Synchronization of SHR}

In users' local network, the section discusses whether the network supports SSM or not and the method how to realize the network synchronization by avoiding timing loops in the path protection ring and the multiplex section protection ring.

SSM of SDH equipments. According to ITU-T, it is stipulated that the S1 byte (b5 b8) in the multiplex section overhead (MSOH) of SDH and Sa4 in E1 signals are considered as SSM to indicate the quality grade of synchronous clocks for current signal flows, as shown in Table 1 . Based on this standard, top-grade clocks are automatically selected as the timing sources, so that the synchronization quality of clocks is furthest improved.

Table 1 SSM coding

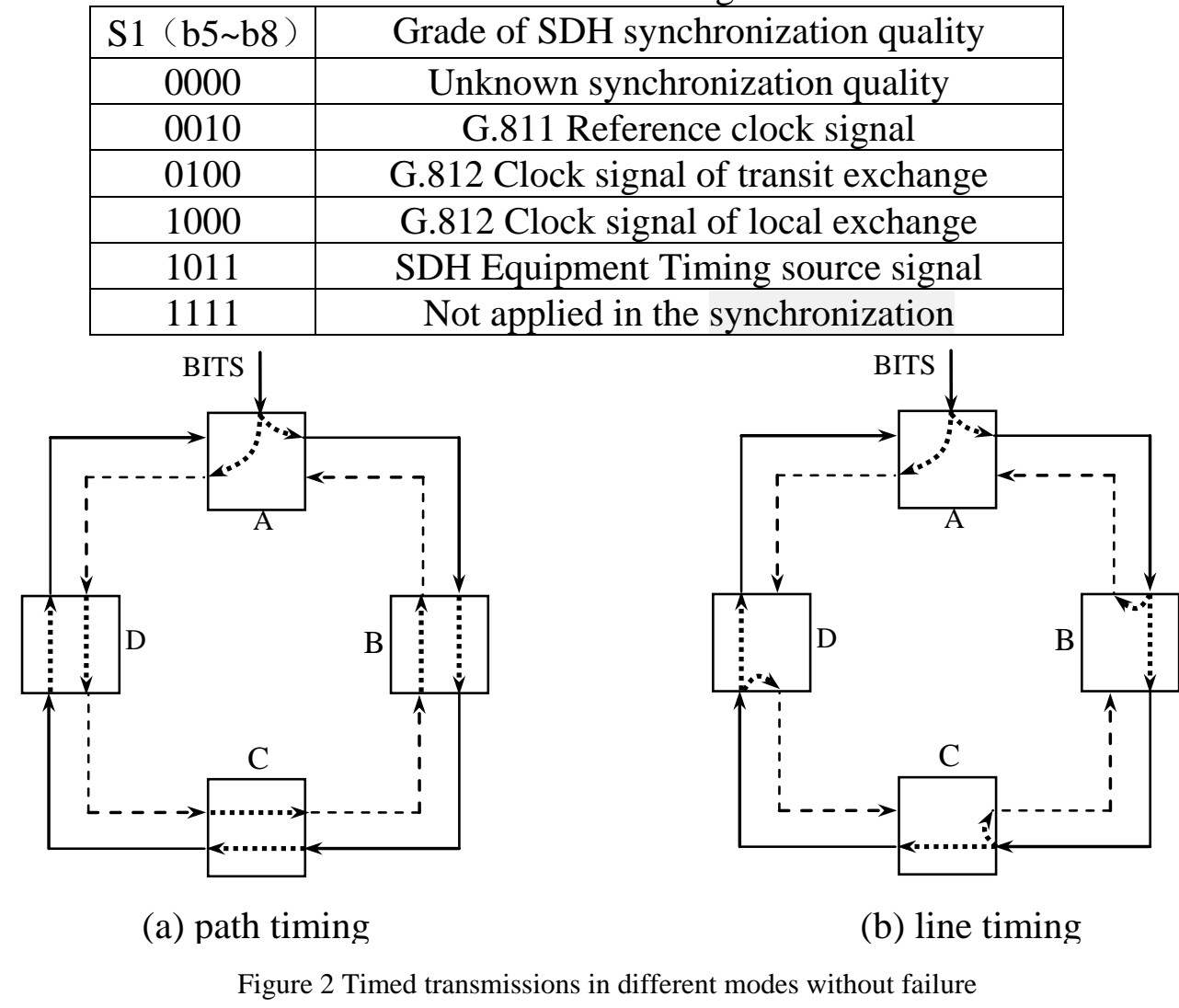

Synchronization of SDH path protection ring. When the path protection ring is applied to the local network, SHR generally has a central node to control the whole timing and external timing is 
commonly performed for this node by the building integrated timing supply (BITS) clock. While other nodes in the ring have to be timed from the central node in line or path timing modes by synchronization transport module -N (STM-N) signal which is transmitted in the line, as illustrated in Figures 2(a) and 2(b).

When the main clock fails, different equipments of these two timing modes are supposed to extract clocks from the input signal in the other direction to serve as the standby ones. Under the condition that SSMs are not supported, when fibers are interrupted, the path protection rings of these two different timing modes switch the timing sources at the nodes at the ends of the failure point, as demonstrated in Figures 3(a) and 3(b).

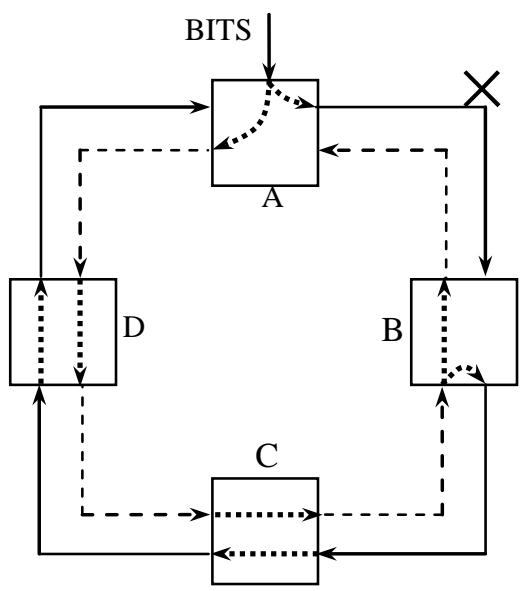

(a) path timing

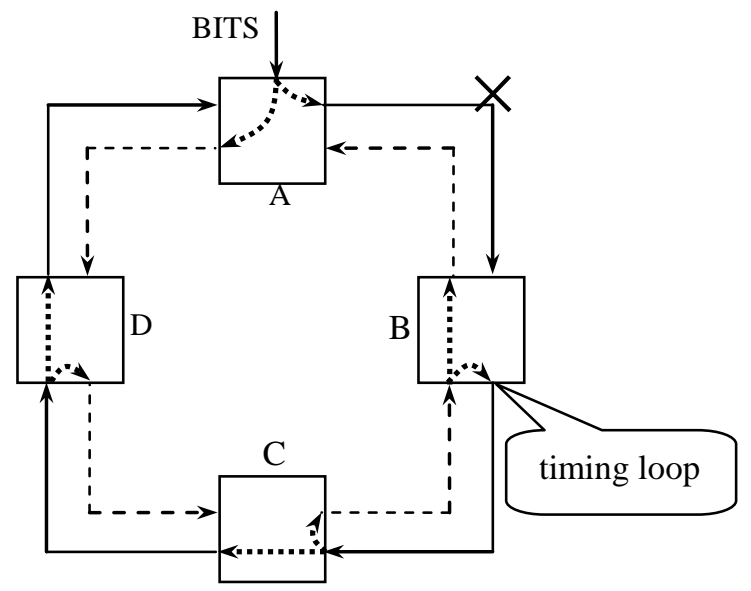

(b) line timing

Figure 3 Timed transmissions in different modes with single failure

From Figures 3 (a) and 3(b), it can be seen that after the fiber between B and C fails, the timing source of the ADM equipment which loses the main clock is expected to be replaced with the standby clock in another direction. No timing loop is formed in the path protection ring of path timing, while timing loops are generated in that of line timing. If failures happen to multiple points, timing loops can also occur in the former. At this moment, network management is needed to avoid the generation of timing loops. Therefore, in the path protection which does not support SSMs, path timing mode is superior to the line timing one.

Synchronization of SDH multiplex section protection ring. Reference timings extracted from the signals in the STM-N line are generally used in the multiplex section protection ring. While once protection switching happens to the multiplex section, the timing loop is likely to be formed if this section does not support SSMs. However, when the timing of the network changes, if SSMs can timely carry timing information, the optimal reference timing can be selected by the network nodes to avoid the generation of timing loops. The author presents a method which can easily realize this function, that is, when the optical cable is broken or a node fails in the multiplex section protection ring, automatic protection switching is performed to the network services using APS protocol which is born by $\mathrm{K} 1$ and $\mathrm{K} 2$ bytes. In the protocol, $\mathrm{K} 1$ is applied as the bridge request to inform others about the failure or force others to conduct the bridge; while $\mathrm{K} 2$ is to confirm the request [4.5]. The arrangement of $\mathrm{K} 1$ and $\mathrm{K} 2$ bytes is illustrated in Figure 4.

It can be known from the arrangement of K1 byte that SF and SD are used to switch the clock source. Modification is merely needed for the software when we use the existing automatic protection switching technology in the equipments. That is, timing loops can be prevented from forming by combining the APS protocol and S1 byte in SSMs. When the equipments are timed in line timing without failures in the network, the information of the transferred $\mathrm{K} 1, \mathrm{~K} 2$ and $\mathrm{S} 1$ bytes in the switching of multiplex section (MS) is displayed in Figure 5. In the condition, K1 byte sends the no-request instructions and the scheduled clock grade information 0100 is filled by the S1 byte to track the reference clock from one direction. Moreover, S1 is applied to fill 1111 in the b5 b8 of S1 
byte of STM-N signal flow in the reverse direction. This information cannot be utilized as the synchronization.

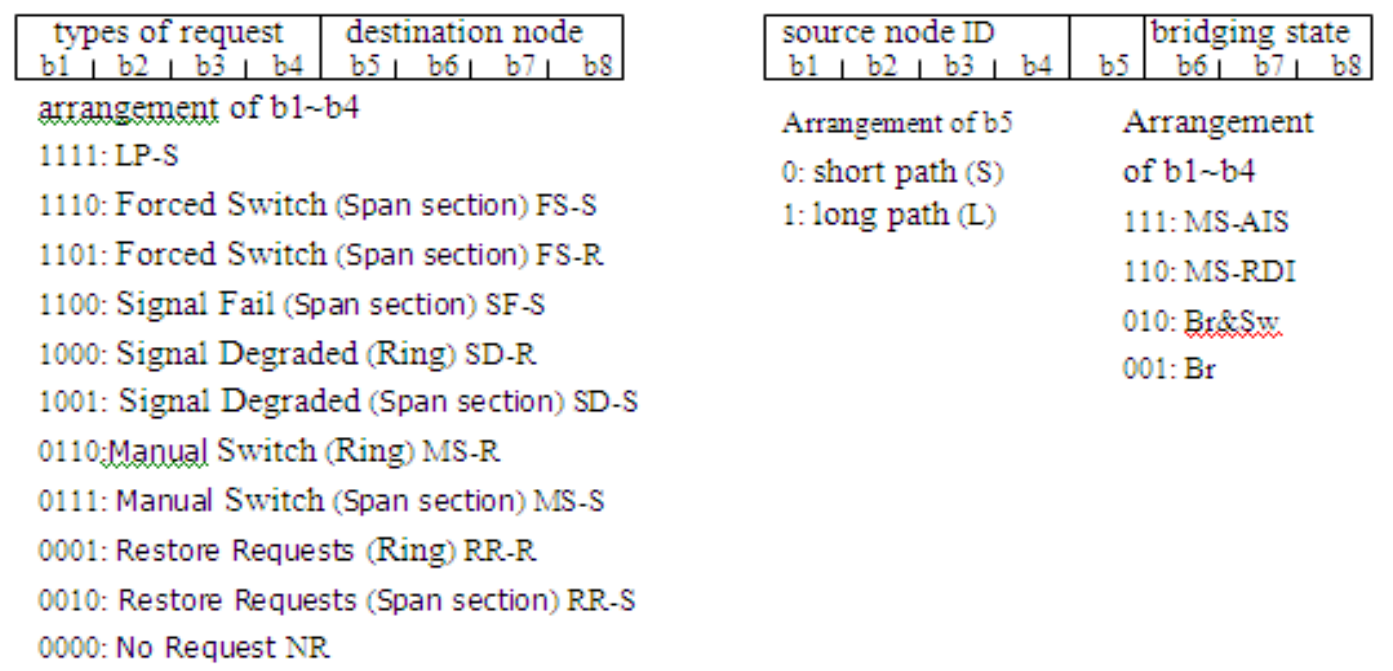

Figure 4 Arrangement of $\mathrm{K} 1$ and $\mathrm{K} 2$ bytes

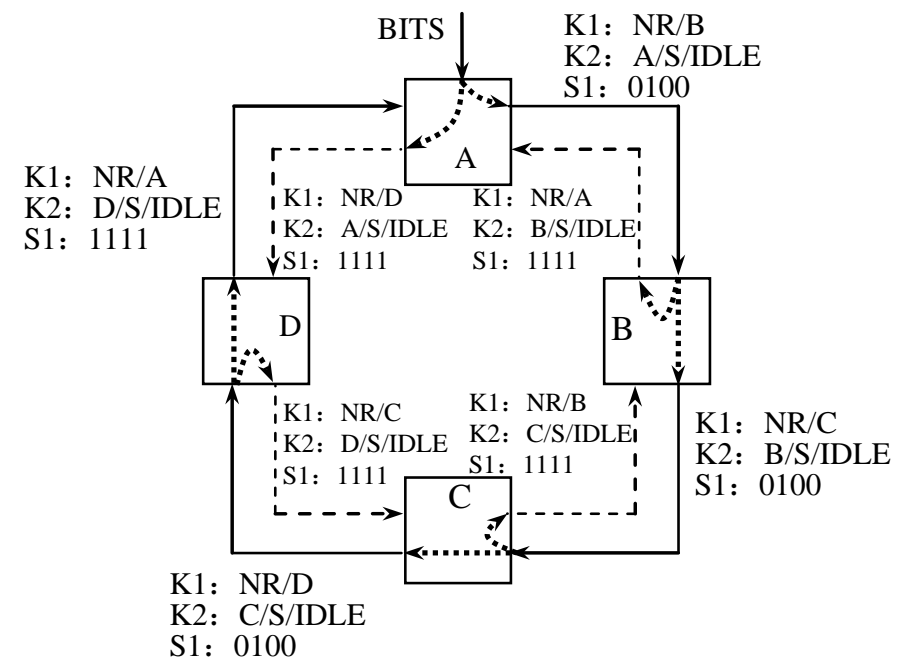

Figure 5 Transmissions of K1, K2 and S1 without failure

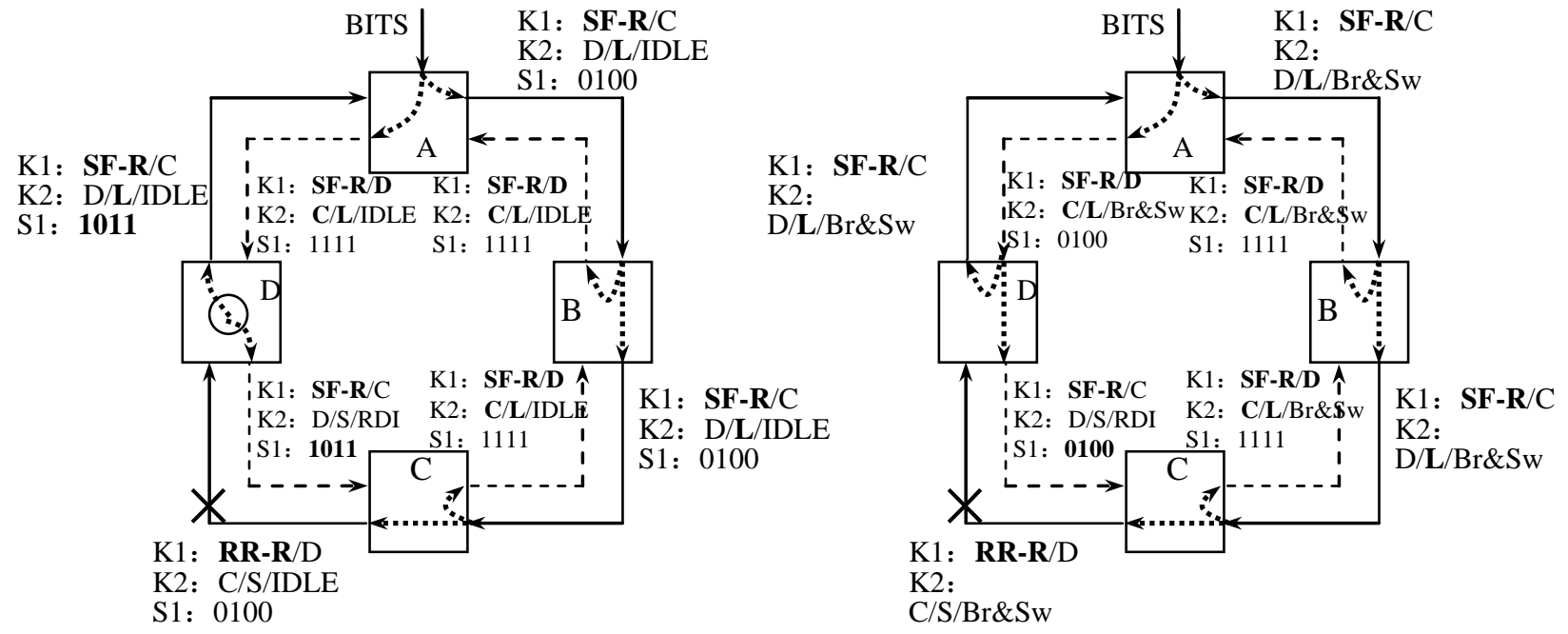

(a) at T1 point

(b) at $\mathrm{T} 2$ point

Figure 6 Transmissions of K1, K2 and S1 with failure 
Figure 6 illustrates the transmissions of $\mathrm{K} 1$ and $\mathrm{K} 2$ bytes in each site after failure happens. Information of failure and bridge request mainly needs to be finished at T1 point. Firstly, when a failure occurs in the D site, signal fail alarming is supposed to be sent from two directions. Then, bridge is applied for the lost information of reference clock contained in the signal failure. At this time, the clock in the D site is remained with 1011 filled in the S1 byte, which is transferred to the adjacent $\mathrm{A}$ site with $\mathrm{K}$ byte. As the $\mathrm{A}$ site receives the $\mathrm{S} 1$ byte sent from the $\mathrm{D}$ site, it shows that the clock of the $\mathrm{D}$ site is remained. Moreover, the information of $\mathrm{K} 1$ and $\mathrm{K} 2$ bytes reflects that the $\mathrm{D}$ site has received the signal failure information of the $\mathrm{C}$ site. According to the obtained information, A site rewrites the information coding 1111 which is not supposed to be synchronous to 1011 which can indicate the quality grade of the clock of the signal flow [6]. While the S1 byte in other sites without failure keeps the same.

At T2 point, $\mathrm{K} 1$ and $\mathrm{K} 2$ bytes transfer the realization of bridge and switching in the $\mathrm{D}$ and $\mathrm{C}$ sites and meanwhile, D site receives the information 0100 of S1 byte from A site to select the STM-N signal flow from the A site as the reference timing. At this time, D site transfers the information of this S1 byte to the following $\mathrm{C}$ site and fills the S1 byte which is fed back to the A site from the D site as 1111, that is, it cannot be applied to the synchronization.

\section{Conclusions}

When timing loops are generated, alarming is generally not sent by the equipments. Therefore at this time, these loops are difficult to be found. Since timing loops present great damage to the network and equipments, they have to be avoided. Based on the above analysis, timing loops can be avoided in the multiplex section protection rings by combining K1 and K2 bytes in APS protocol with S1 byte. In this way, synchronization performance can be improved.

\section{Acknowledgement}

In this paper, the research was sponsored by key project of education department in Shaanxi provincial government (Project No. 13BZ69)

\section{References}

[1] Wu Wei-jing. The Discuss on the Loop of SDH Ring-forming System Timepiece[J]. Manufacture Information Engineering of China, 2005(09):105-106.

[2] Han Jing, Lu Hai-jun, Yuan Xin-chang. The demand on the SDH transmission timing of New businesses [J].Modern Cable transimission, 1999(04):25-28.

[3] Sun Jian-feng. The Study on synchronization Performance Application of Clock and SDH network [J]. Inner Mongolia Scinece Technology \& Economy, 2014(01): 65-67.

[4] Li Xiao-zhen, Su Jian-feng. The Study on High-accuracy Network Clock Synchronization Based on IEEE1588[J]. Communications Technology, 2011,44(03):105-108.

[5] Song Bo,Sun Chao,Jiang Shou-da. A High-Precision Local Area Network Clock Synchronization Method Based on PTP Protocol [J], 2010,29(01):67:74.

[6] Li Xue-qiao, Chen Yuan,Liang, Shuang. Improvement of the Precise Time Synchronization Algorithm Based on IEEE1588 [J], 2011, 26(02):42:45. 